\title{
Gli antagonisti del recettore GPIIb/IIIa: farmacologia, clinica ed economia nelle sindromi coronariche acute NSTEMI e nelle rivascolarizzazioni per via percutanea
}

Lorenzo Pradelli*

\begin{abstract}
Inhibition of platelet glycoprotein IIb/IIIa (GP IIb/IIIa) receptor prevents platelet aggregation by controlling its final common pathway, the cross-binding of fibrinogen, bridging across adjacent platelets. Three pharmacological agents capable of inhibiting GP IIb/IIIa are available for use in Italy: abciximab, eptifibatide and tirofiban. In this paper, some relevant studies on the pharmacology of GP IIb/IIIa inhibitors are summarized, as well as the main clinical trials assessing their use in the management of unstable angina (UA) and during percutaneous coronary interventions (PCI). Furthermore, the recommendations on their appropriate use in UA and PCI issued by authoritative scientific societies are presented. Finally, some of the pharmacoeconomic evidence published in the international literature is reviewed and implications in the Italian health care setting are discussed.
\end{abstract}

Keywords: GP IIb/IIIa inhibitors, unstable angina, percutaneous coronary interventions Farmeconomia e percorsi terapeutici 2005; 6 (4): 305-316

\section{INTRODUZIONE}

L'aggregazione piastrinica rappresenta la tappa finale del processo che porta alla formazione del tappo piastrinico e pone le premesse per l'attivazione del sistema della coagulazione, con attivazione della trombina e formazione del coagulo di fibrina. Il meccanismo biochimico che permette alle piastrine attivate di legarsi fra loro è rappresentato dall'esposizione dei complessi glicoproteici GPIIb-IIIa, che si comportano da recettori del fibrinogeno. Il fibrinogeno legato ai recettori di piastrine adiacenti forma dei veri e propri ponti tra piastrina e piastrina e permette la formazione degli aggregati piastrinici.

Nelle sindromi coronariche acute senza sopraslivellamento del tratto ST (angina instabile e NSTEMI o infarti non-Q) e negli interventi di angioplastica per via percutanea (PCI), l'inibizione farmacologica del legame tra GPIIbIIIa e fibrinogeno (ma anche fattore von Willebrand e altri ligandi molecolari), associata all'azione antitrombotica dell'acido acetilsalicilico e dell'eparina, è in grado di ridurre il rischio di occlusione dei vasi interessati e, di conseguenza, di ridurre l'incidenza di morte, infarto e di re-intervento di rivascolarizzazione. I tre agenti farmacologici in grado di inibire i recettori GPIIb-IIIa attualmente disponibili sono abciximab, eptifibatide e tirofiban, ma il loro utilizzo, a dispetto della comprovata efficacia, è ancora piuttosto limitato, come evidenziato dai risultati dei Registri Europei e Americani.

\section{FARMACOLOGIA}

Gli antagonisti del recettore glicoproteico (GP), per quanto differenti dal punto di vista strutturale e farmacologico, inibiscono in modo selettivo la via finale comune dell'aggregazione piastrinica, cioè il legame del fibrinogeno alla superficie della piastrina del recettore GP. Per ottenere una rapida e duratura inibizione dell'aggregazione piastrinica (il livello di inibizione dell'attività piastrinica necessario per avere una efficace risposta clinica deve essere superiore all' $80 \%$ ), gli antagonisti GP IIb/IIIa sono somministrati sotto forma di uno o due boli endovenosi, seguiti da una somministrazione in infusione endovenosa lenta.

Abciximab è un anticorpo monoclonale chimerico (regione costante umana - Fab mu- 
rino) specifico per i recettori GP IIb-IIIa. L'inibizione della funzionalità piastrinica è in genere evidente poco dopo la somministrazione e non più rilevabile dopo 48 ore dalla sua sospensione, per quanto abciximab rimanga nella circolazione sanguigna anche per 10 giorni, legato alle piastrine $[1,2]$.

Tirofiban è un derivato della tirosina che agisce da antagonista selettivo dei recettori IIb/IIIa; la funzionalità piastrinica è rapidamente e significativamente ridotta dopo iniezione endovenosa e tale inibizione è mantenuta per 3-8 ore dal termine dell' infusione $[3,4]$.

Eptifibatide è un eptapeptide ciclico che antagonizza i recettori GP IIb/IIIa in maniera selettiva, reversibile e dose- e concentrazionedipendente. La funzionalità piastrinica è significativamente ridotta entro 10 minuti dalla somministrazione e ritorna a valori normali entro 2 4 ore dalla sua sospensione [2,5-8].

Il profilo farmacodinamico di questi tre agenti è stato direttamente confrontato in uno studio randomizzato in doppio cieco [5], condotto su 30 pazienti sottoposti a rivascolarizzazione per via percutanea (PCI), in cui il livello e l'andamento temporale dell'inibizione dell'aggregabilità piastrinica è stata valutata ex vivo con due differenti metodiche, l'aggregometria ottica e un kit per la valutazione rapida della funzionalità piastrinica. I pazienti hanno ricevuto uno dei 3 farmaci secondo la posologia approvata: abciximab $0,25 \mathrm{mg} /$ $\mathrm{kg}$ come bolo ev, seguito da infusione ev continua di $0,125 \mu \mathrm{g} / \mathrm{kg} / \mathrm{min}$ per 12 ore; tirofiban $0,4 \mu \mathrm{g} / \mathrm{kg}$ per 30 minuti come bolo, seguito da infusione continua di $0,1 \mu \mathrm{g} / \mathrm{kg} / \mathrm{min}$ per $20-24$ ore; eptifibatide $180 \mu \mathrm{g} / \mathrm{kg}$ come bolo ev, seguita da infusione continua di $2,0 \mu \mathrm{g} / \mathrm{kg} / \mathrm{min}$ per 20-24 ore. Le due metodologie hanno fornito risultati sostanzialmente concordanti, che si possono così sintetizzare:

- l'intensità desiderata di inibizione della funzionalità piastrinica (> 80\% secondo il test del kit rapido) viene raggiunta rapidamente (entro 10 minuti) dopo il bolo di abciximab ed eptifibatide, mentre per tirofiban la latenza tra inizio della somministrazione e il raggiungimento di un'efficace inibizione dell' aggregabilità è maggiore, probabilmente a causa della differente strategia utilizzata per il bolo "di carico";

- l'inibizione dell'aggregazione piastrinica raggiunta è stata minore con il tirofiban rispetto a abciximab o eptifibatide: tirofiban ha mostrato una riduzione media $>80 \%$ all'aggregometria a trasmissione ottica solo alla misurazione effettuata a 18 ore, mentre questo livello di attività è stato sempre raggiunto dagli altri due farmaci;

- l'intensità dell' attività antiaggregante piastrinica di abciximab diventa meno uniforme e costante prima della sospensione della somministrazione, probabilmente a causa dei bassi livelli plasmatici di farmaco libero, che potrebbero non essere sufficienti a bloccare tutti i recettori GPIIb/IIIa liberati dai granuli alfa delle piastrine attivate [5].

La farmacodinamica delle tre molecole è stata inoltre valutata in studi specifici di confronto. Nello studio TAM1 l'attività piastrinica è stata valutata in 40 pazienti sottoposti a PCI e trattati con tirofiban, somministrato con lo schema posologico degli studi TARGET e RESTORE (bolo di $10 \mu \mathrm{g} / \mathrm{kg}$ seguito da infusione continua di $0,15 \mu \mathrm{g} / \mathrm{kg} / \mathrm{min}$ per 18 - 24 ore) o con eptifibatide somministrata secondo lo schema posologico del trial ESPRIT (bolo di $180 \mu \mathrm{g} / \mathrm{kg}$, seguito da infusione continua di 2 $\mu \mathrm{g} / \mathrm{kg} / \mathrm{min}$ per $18-24$ ore e da un ulteriore bolo di $180 \mu \mathrm{g} / \mathrm{kg} 10$ minuti dopo il primo). I risultati dello studio TAM1 indicano che l'inibizione dell'aggregazione piastrinica è raggiunta più precocemente con eptifibatide $(\mathrm{p}=0,003)$ e che il livello medio di inibizione è significativamente superiore a quello ottenuto con tirofiban per tutta la durata dell'infusione $(\mathrm{p}<0,0001)$. Inoltre, pressoché tutti i pazienti assegnati a eptifibatide hanno mostrato livelli di inibizione target (cioè superiore all' $80 \%$ ) per l'intera durata dell'infusione, mentre i pazienti trattati con tirofiban hanno raggiunto un livello medio di inibizione inferiore all' $80 \%$ a 15 e 30 minuti dal bolo, e una quota non trascurabile di pazienti ha mostrato livelli di inibizione $\leq 80 \%$ per tutta la durata dell'infusione [8].

Nello studio TAM2, eptifibatide somministrata con lo schema posologico utilizzato nello studio PURSUIT (bolo di $180 \mu \mathrm{g} / \mathrm{kg}$, seguito da infusione continua di $2 \mu \mathrm{g} / \mathrm{kg} / \mathrm{min}$ per almeno 24 ore) è stata confrontata con abciximab, somministrato con lo schema posologico del trial GUSTO IV (bolo di $0,25 \mathrm{mg} / \mathrm{kg}$, seguito da infusione continua di $0,125 \mu \mathrm{g} / \mathrm{kg} / \mathrm{min}$ per almeno 24 ore) in 40 pazienti con sindrome coronarica acuta. Il livello medio di inibizione dell'aggregazione piastrinica è stato costantemente superiore all' $80 \%$ con eptifibatide per l'intera durata dell'infusione e significativamente maggiore di quello ottenuto con abciximab ( $\mathrm{p}<0,0001)$. L'attività di abciximab, come in altri studi di farmacodinamica, è diminuita e ha mostrato un elevato grado di variabilità interindividuale dopo qualche ora dall'inizio dell'infusione: il livello medio di inibizione è risultato $<80 \%$ a $6,8,12,18$ e 24 ore [2].

\section{COLLOCAZIONE TERAPEUTICA DEGLI INIBITORI GPIIB/IIIA}

\section{Trattamento upstream}

\section{Eptifibatide}

Nel trial PURSUIT (Platelet Glycoprotein IIb-IIIa in Unstable Angina: Receptor 
Suppression Using Integrilin Therapy) ( $\mathrm{n}=10.948$ ), eptifibatide (bolo di $180 \mu \mathrm{g} / \mathrm{kg}$ seguito da un'infusione di $2 \mu / \mathrm{kg} /$ minuto) ha significativamente ridotto l'incidenza dell'endpoint composito (morte o infarto miocardico non letale) rispetto al placebo, cioè la terapia standard $(14,2 \%$ versus $15,7 \%$; $\mathrm{p}=0,04$; riduzione assoluta d'incidenza $1,5 \%$ ). La maggioranza dei pazienti ha ricevuto inoltre acido acetilsalicilico (ASA) 75 - $325 \mathrm{mg}$ uid ed eparina a discrezione del ricercatore, più comunemente come bolo endovenoso di 5000 unità seguito da infusione continua di 12 unità/kg/ora. Nei pazienti trattati con eptifibatide e sottoposti a PCI entro 72 ore dalla randomizzazione si è ottenuta una riduzione del $31 \%$ dell'endpoint composito a 30 giorni, rispetto a quelli trattati con placebo (11,6\% versus $16,7 \%$, $\mathrm{p}=0,01)$. La riduzione di incidenza degli eventi è stata ottenuta a 96 ore e mantenuta a 7, $30 \mathrm{e}$ 180 giorni di follow-up [9-12].

In ragione del diverso accesso alle risorse, in particolare all'utilizzo più estensivo della coronarografia diagnostica e della rivascolarizzazione sia meccanica sia chirurgica, era stato pre-identificato il gruppo dei pazienti arruolati negli USA. L'analisi condotta su questo gruppo di pazienti, conosciuto come PURSUITUS, ha rivelato che l'incidenza dell'endpoint primario costituito da infarto e morte a 30 giorni è risultata ridotta dal $15,4 \%$ del gruppo placebo $(n=1766)$ all'11,9\% del gruppo eptifibatide $(\mathrm{n}=1756)$ (riduzione assoluta del $3,5 \% ; \mathrm{p}=0,0025)$ e tale effetto è stato mantenuto per un periodo di 6 mesi $(18,9 \%$ versus $15,2 \% ; p=0,004)$ [13].

Sempre nel PURSUIT, l'esecuzione precoce (entro 24 ore dalla randomizzazione) di una PCI associata a concomitante trattamento con eptifibatide è risultata in esiti clinici significativamente migliori rispetto a PCI eseguite successivamente o senza inibitore GPIIb/IIIa. L'incidenza cumulativa di infarto o morte a 30 giorni è stata del 9,2\% nei pazienti rivascolarizzati per via percutanea nel primo giorno $(n=620)$, del $14 \%$ in quelli con PCI eseguita in seconda e terza giornata $(n=624)$, del $15 \%$ tra 4 e 7 giorni $(n=614)$ e $17,4 \%$ tra 8 e 30 giorni $(n=561)$. Nei pazienti con PCI eseguita nelle prime 24 ore, ma senza concomitante eptifibatide, l'incidenza è stata del $15,9 \%(\mathrm{p}=0,011$ versus eptifibatide con PCI). Gli autori di quest' analisi sottolineano che i loro risultati suggeriscono che una strategia conservativa di "vigile attesa" delle sindromi coronariche acute sia inferiore a un atteggiamento più aggressivo [14].

Un' analisi retrospettiva ha mostrato che tra i pazienti sottoposti a chirurgia coronarica entro due ore dalla sospensione di eptifibatide la frequenza di eventi emorragici non è risultata significativamente diversa da quella osservata nei pazienti randomizzati a placebo, mentre $\mathrm{i}$ risultati clinici sono migliorati: l'incidenza di infarto del miocardio a 30 giorni è risultata dimezzata $(46 \%$ versus $22 \%, \mathrm{p}=0,035)$. La trombocitopenia e la riduzione assoluta della conta piastrinica sono state minori nel gruppo eptifibatide $(p=0,04)$, suggerendo la presenza un effetto di risparmio sul consumo di trombociti. Il $98 \%$ e il $96 \%$ dei pazienti dei gruppi placebo ed eptifibatide, rispettivamente, hanno ricevuto eparina; tutti i pazienti erano anche in terapia con ASA [15].

La dose di eptifibatide utilizzata nel PURSUIT è stata maggiore di quella adottata in studi precedenti, in quanto inizialmente la capacità disaggregante del farmaco era stata sovrastimata perché il metodo di analisi utilizzato per la determinazione della dose efficace non era appropriato [16]. Di conseguenza i risultati clinici del PURSUIT sono migliori di quelli osservati precedentemente [17].

\section{Tirofiban}

Nello studio PRISM-PLUS (Platelet Receptor Inhibition in Ischemia Syndrome Management in Patients Limited by Unstable Signs and symptoms), condotto su pazienti con sindrome coronarica acuta, l'associazione di tirofiban ed eparina è risultata più efficace nel prevenire eventi ischemici rispetto all'eparina in monoterapia; entrambi i trattamenti sono stati associati a ASA $325 \mathrm{mg} / \mathrm{die}$. Inizialmente $\mathrm{i}$ pazienti erano stati suddivisi in tre gruppi di trattamento: tirofiban in monoterapia $(0,6 \mu \mathrm{g} / \mathrm{kg} / \mathrm{min}$ per 30 minuti, seguiti dall'infusione di $0,15 \mu \mathrm{g} / \mathrm{kg} / \mathrm{min}+$ placebo simil-eparina), tirofiban ed eparina in associazione (tirofiban $0,4 \mu \mathrm{g} / \mathrm{kg} / \mathrm{min}$ per 30 minuti seguiti da $0,1 \mu \mathrm{g} / \mathrm{kg} / \mathrm{min}$; eparina bolo $5000 \mathrm{UI}$ seguiti da $1000 \mathrm{UI} /$ ora, aggiustate a 6, 12, 24, 36 e 48 ore per raggiungere un APTT 2 volte superiore al valore di controllo) ed eparina in monoterapia (stessa dose del gruppo precedente + placebo simil-tirofiban). L'infusione dei farmaci in studio è stata mantenuta per almeno 48 ore e ogni intervento è stato posticipato oltre tale limite, se possibile. L'angiografia e/o l'angioplastica coronariche sono state effettuate, quando indicate, dopo 48 -96 ore di infusione, mantenuta anche durante la procedura di rivascolarizzazione. Tirofiban in monoterapia è stato sospeso per inefficacia, in seguito all'osservazione di un eccesso di mortalità nell'analisi a 7 giorni $(4,6 \%$ - 16 di 345 - vs $1,1 \%$ - 4 di 350 - del gruppo eparina in monoterapia e $1,5 \%$ - 5 di 336 - del gruppo tirofiban + eparina). L'incidenza dell'endpoint composito (morte, re-infarto, ischemia refrattaria, riospedalizzazione per angina instabile) nei gruppi eparina in monoterapia e tirofiban più eparina è stata di $7,8 \%$ e $5,7 \%$ a 48 ore $(\mathrm{p}=$ $0,08), 17,9 \%$ e $12,9 \%$ a 7 giorni $(p=0,004), 22,3 \%$ 
e $18,5 \%$ a 30 giorni ( $\mathrm{p}=0,03 \mathrm{NS})$, e di $32,1 \%$ e $27,7 \%$ a 6 mesi $(p=0,02)$, rispettivamente [19].

Lo studio PRISM (Platelet Receptor Inhibition in Ischemic Syndrome Management) ha confrontato tirofiban $(0,6 \mu \mathrm{g} / \mathrm{kg} / \mathrm{min}$ per 30 minuti seguiti da $0,15 \mu \mathrm{g} / \mathrm{kg} / \mathrm{min})$ ed eparina entrambi somministrati per via endovenosa, in 3.232 pazienti con segni di ischemia miocardica.

L'incidenza dell' endpoint composito (morte, infarto, ischemia refrattaria) è risultata significativamente ridotta a 48 ore $(3,8$ versus $5,6 \%, \mathrm{p}=0,01)$, ma non a 7 e 30 giorni, nonostante la riduzione della mortalità complessiva con tirofiban abbia mostrato un trend al limite della significatività $(2,3$ vs $3,6 \%, p=0,02)$ [19].

Alcune differenze tra i due studi possono spiegare la notevole discrepanza tra i loro risultati: nel PRISM-PLUS, rispetto al PRISM, le alterazioni dei tratti ST o ST-T all'ECG erano molto più frequenti $(90 \%$ vs $39 \%)$, la durata dell'infusione di tirofiban maggiore ( 71 vs 48 ore), l'angiografia o angioplastica durante l'infusione di tirofiban non era scoraggiata dal protocollo di studio e i pazienti hanno ricevuto eparina prima della randomizzazione [20].

\section{Abciximab}

Nello studio GUSTO-IV ACS $(\mathrm{n}=7800)$, condotto su pazienti con sindrome coronarica acuta non rivascolarizzati precocemente, la somministrazione di abciximab in upstream non è risultata associata a benefici clinici maggiori del placebo.

I criteri di inclusione dello studio prevedevano la presenza di uno o più episodi di angina di durata maggiore di 5 minuti nelle 24 ore precedenti e un altro segno di ischemia miocardica (test positivo per la troponina o depressione del tratto ST di almeno $5 \mathrm{~mm}$ ); i soggetti sono stati suddivisi in tre gruppi di trattamento: abciximab per 24 ore $(0,25 \mathrm{mg} /$ $\mathrm{kg}$ in bolo, seguito da $0,125 \mu \mathrm{g} / \mathrm{kg} / \mathrm{min}$, fino a un massimo di $10 \mu \mathrm{g} / \mathrm{min}$, per 24 ore) $(n=2590)$, abciximab 48 ore (stesse dosi precedenti, ma infusione mantenuta per 48 ore) $(n=2612)$ e placebo $(n=2598)$; tutti i pazienti hanno ricevuto ASA ed eparina o dalteparina.

L'endpoint primario di morte o infarto a 30 giorni si è verificato con frequenza simile nei tre gruppi $(8,2 \%, 9,1 \%, 8 \%$ per abciximab 24 ore, abciximab 48 ore e placebo, rispettivamente; rischio relativo 1,0 per abciximab 24 ore vs placebo e di 1,1 per abciximab 48 ore vs placebo).

Si è inoltre osservato un aumento della mortalità nel gruppo di pazienti trattati con abciximab per 48 ore rispetto al placebo. La valutazione dell'endpoint di morte o infarto miocardico a 30 giorni nei sottogruppi (per sesso, età, presenza di diabete, troponine elevate, depressione ST) non ha evidenziato alcun vantaggio della somministrazione di abciximab in upstream rispetto al placebo [21].

\section{Efficacia nelle PCI}

\section{Eptifibatide}

Lo studio ESPRIT (Enhanced Suppression of the Platelet IIb/IIIa Receptor with Integrilin Therapy) ha dimostrato che eptifibatide, somministrato come bolo endovenoso di $180 \mu \mathrm{g} /$ $\mathrm{kg}$, seguito immediatamente da infusione continua di $2 \mu \mathrm{g} / \mathrm{kg} / \mathrm{min}$ e da un secondo bolo di $180 \mu \mathrm{g} / \mathrm{kg}$ con infusione mantenuta per $18-24$ ore, migliora significativamente gli esiti dopo stenting coronarico d'elezione. Questo studio randomizzato in doppio cieco e multicentrico $(n=2064)$ è stato interrotto precocemente per dimostrata efficacia di eptifibatide: l'endpoint primario composito (morte, infarto, rivascolarizzazione del vaso trattato o terapia antitrombotica bailout entro 48 ore) è stato ridotto dal $10,5 \%$ (108 di 1024) dei pazienti trattati con placebo al 6,6\% (69 di 1040) di quelli che hanno ricevuto il farmaco ( $R R$ 0,63; $\mathrm{p}=0,0015$ ). A 30 giorni dall'intervento, l'endpoint secondario di morte, infarto o rivascolarizzazione del vaso trattato ha dimostrato una simile riduzione di incidenza (10,5\% placebo versus $6,8 \%$ eptifibatide; RR 0,65; $\mathrm{p}=0,0034)$.Oltre l' $88 \%$ di tutti gli eventi (157 di 178) si sono verificati nelle prime 48 ore. Tutti i pazienti erano stati pre-trattati con ASA, una tienopiridina (principalmente clopidogrel) ed eparina. Si è osservato un leggero aumento di incidenza di emorragie $(1,3 \%$ vs $0,4 \mathrm{p}=0,027)$ in particolare nei pazienti con APTT elevato e trattati con eptifibatide [22].

I risultati dei follow-up di ESPRIT a 6 e 12 mesi confermano che nei pazienti sottoposti a stenting coronarico programmato la terapia con eptifibatide è associata a benefici clinicamente significativi anche nel lungo termine. A 180 giorni, l'endpoint composito di morte, infarto o rivascolarizzazione del vaso trattato è stato osservato nel $18,3 \%$ dei pazienti placebo rispetto al $14,2 \%$ dei trattati con eptifibatide $(\mathrm{p}=0,008$, RR 0,75); l'incidenza di infarto o morte è stata dell' $11,5 \%$ nel gruppo placebo e del $7,5 \%$ nel gruppo eptifibatide $(\mathrm{p}=0,002, \mathrm{RR} 0,63)$ [23]. A 1 anno dall'intervento ( $\mathrm{n}=976$ placebo, 988 eptifibatide), l'endpoint triplo si è verificato nel $17,5 \%$ e nel $22,1 \%$ (RR 0,76; $\mathrm{p}=0,007$ ) dei pazienti eptifibatide e placebo, rispettivamente, mentre l'incidenza di infarto o morte è stata del 8,0\% e del 12,4\% (RR 0,63; p=0,001). Questa riduzione è da attribuire verosimilmente alla riduzione degli infarti nelle prime 48 ore (IMA a 48 ore $9,0 \%$ vs $5,4 \%, p=0,0015$ ), e il beneficio acquisito si è mantenuto per almeno un anno (Tabella I). 


\begin{tabular}{|c|c|c|c|c|c|c|c|c|}
\hline \multirow{2}{*}{$\begin{array}{c}\text { Outcome } \\
\text { Tempo di } \\
\text { osservazione }\end{array}$} & \multicolumn{4}{|c|}{$\begin{array}{c}\text { Morte, infarto o rivascolarizzazione } \\
\text { del vaso sottoposto a } \mathrm{PCl}\end{array}$} & \multicolumn{4}{|c|}{ Morte o infarto } \\
\hline & 48 ore & $30 \mathrm{gg}$ & 6 mesi & 1 anno & 48 ore & $30 \mathrm{gg}$ & 6 mesi & 1 anno \\
\hline $\begin{array}{l}\text { Placebo } \\
(n=1024)\end{array}$ & $\begin{array}{c}95 \\
(9,3 \%)\end{array}$ & $\begin{array}{c}107 \\
(10,4 \%)\end{array}$ & $\begin{array}{c}187 \\
(18,5 \%)\end{array}$ & $\begin{array}{c}222 \\
(22,1 \%)\end{array}$ & $\begin{array}{c}94 \\
(9,2 \%)\end{array}$ & $\begin{array}{c}104 \\
(10,2 \%)\end{array}$ & $\begin{array}{c}117 \\
(11,5 \%)\end{array}$ & $\begin{array}{c}126 \\
(12,4 \%)\end{array}$ \\
\hline $\begin{array}{l}\text { Eptifibatide } 180 / 2,0 / 180 \\
(n=1040)\end{array}$ & $\begin{array}{c}62 \\
(6,0 \%)\end{array}$ & $\begin{array}{c}71 \\
(6,8 \%)\end{array}$ & $\begin{array}{c}146 \\
(14,3 \%)\end{array}$ & $\begin{array}{c}178 \\
(17,5 \%)\end{array}$ & $\begin{array}{c}57 \\
(5,5 \%)\end{array}$ & $\begin{array}{c}66 \\
(6,3 \%)\end{array}$ & $\begin{array}{c}77 \\
(7,4 \%)\end{array}$ & $\begin{array}{c}83 \\
(8,0 \%)\end{array}$ \\
\hline $\begin{array}{l}\text { Riduzione assoluta } \\
\text { del rischio }\end{array}$ & $3,3 \%$ & $3,6 \%$ & $4,2 \%$ & $4,6 \%$ & $3,7 \%$ & $3,9 \%$ & $4,1 \%$ & $4,4 \%$ \\
\hline $\begin{array}{l}\text { Rischio Relativo } \\
\text { IC 95\% } \\
\text { p }\end{array}$ & $\begin{array}{c}0,643 \\
0,472-0,875 \\
0,0045\end{array}$ & $\begin{array}{c}0,653 \\
0,490-0,871 \\
0,0034\end{array}$ & $\begin{array}{l}0,744 \\
0,599 \\
0,924\end{array}$ & $\begin{array}{l}0,762 \\
0,626 \\
0,929\end{array}$ & $\begin{array}{c}0,6 \\
0,435-0,820 \\
0,0013\end{array}$ & $\begin{array}{c}0,625 \\
0,465-0,840 \\
0,0016\end{array}$ & $\begin{array}{l}0,631 \\
0,473 \\
0,841\end{array}$ & $\begin{array}{l}0,630 \\
0,478 \\
0,832\end{array}$ \\
\hline
\end{tabular}

Tabella I

Risultati a breve e lungo termine osservati nello studio ESPRIT

Come accennato in precedenza, in studi precedenti all'ESPRIT, l'effetto antiaggregante di eptifibatide era stato sovrastimato per l'utilizzo di una metodica inadeguata di determinazione dell'effetto antiaggregante [16,17], per cui sono stati utilizzati dosaggi inferiori, con corrispondente riduzione dell' ampiezza del beneficio clinico ottenuto. Nell'IMPACT II (Integrilin to Minimize Platelet Aggregation and Coronary Thrombosis) $(\mathrm{n}=4010)$, ad esempio, la somministrazione di un bolo di $135 \mu \mathrm{g} / \mathrm{kg}$, seguito da un'infusione di $0,5 \mu \mathrm{g} / \mathrm{kg} / \mathrm{min}$, in pazienti assegnati a PCI è risultata associata a una riduzione minore, benché statisticamente significativa $(11,6 \%$ versus $9,1 \%, \mathrm{p}=0.035)$, dell'endpoint primario, costituito dal tasso complessivo di mortalità, infarto miocardico o intervento coronarico non programmato [24].

\section{Tirofiban}

Lo studio RESTORE (Randomized Efficacy Study of Tirofiban for Outcomes and REstenosis) [25] è stato condotto con l'obiettivo di dimostrare che tirofiban, somministrato come bolo di $10 \mu \mathrm{g} / \mathrm{kg}$ seguito da un'infusione continua di $0,15 \mu \mathrm{g} / \mathrm{kg} / \mathrm{min}$ per 36 ore, è in grado di ridurre significativamente gli eventi cardiovascolari (morte, IMA, CABG, re-intervento di coronoplastica o stenting) a 30 giorni rispetto al placebo in pazienti con sindrome coronarica acuta sottoposti a PCI entro 72 ore dall'esordio dei sintomi. Benché l'incidenza dell'endpoint a 30 giorni sia risultata ridotta del $1,9 \%(12,2 \%$ vs $10,3 \%)$ nei pazienti trattati con tirofiban, tale differenza non ha raggiunto la significatività statistica $(\mathrm{p}=0,16)$.

In uno studio di fase II, randomizzato in doppio cieco e controllato versus placebo (ADVANCE, $n=202$ ), tirofiban somministrato con un regime posologico comprendente un bolo ad alto dosaggio $(25 \mu \mathrm{g} / \mathrm{kg})$ e un'infusione di $0,15 \mu \mathrm{g} / \mathrm{kg} / \mathrm{min}$ della durata di $24-48$ ore, ha ridotto significativamente la mortalità e gli eventi ischemici in pazienti ad elevato rischio sottoposti a PCI (con stenting nel $98 \%$ dei casi).
L'endpoint primario (morte, infarto, rivascolarizzazione del vaso trattato o terapia bailout) valutato a 180 giorni, si è verificato nel $20 \%$ dei pazienti trattati con tirofiban e nel $35 \%$ dei pazienti assegnati al placebo $(\operatorname{RR} 0,51 ; \mathrm{p}=0,01)$. La riduzione endpoint combinato è stata in gran parte dovuta alla riduzione della componente degli infarti miocardici; non è stato osservato alcun effetto significativo sulla mortalità o sulla necessità di re-intervenire sul vaso target [26]. Si attendono ulteriori studi che confermino l'efficacia di questo dosaggio in una popolazione più numerosa, tali da consentire una corretta valutazione farmacoeconomica.

\section{Abciximab}

L'utilizzo di abciximab in associazione allo stenting è stato valutato in numerosi studi; in generale, appare che il trattamento con abciximab sia in grado di ridurre significativamente gli eventi ischemici nei pazienti ad alto rischio, mentre in soggetti con un profilo di rischio più moderato la sua utilità è risultata meno evidente.

In EPILOG, uno studio randomizzato condotto su 2792 pazienti sottoposti a PCI d'urgenza o programmata, i pazienti inclusi nei due bracci di trattamento (abciximab + eparina a basse dosi e abciximab + eparina a dosi standard) hanno mostrato esisti clinici significativamente superiori a quelli del gruppo di controllo: l'incidenza a 30 giorni endpoint combinato (morte, infarto, rivascolarizzazione non programmata) è stata dell' $11,7 \%, 5,2 \%$ e $5,4 \%$ nei gruppi placebo, abciximab + eparina a basse dosi e abciximab + eparina standard, rispettivamente $(\mathrm{p}<0,05$ per $\mathrm{i}$ due gruppi attivi vs placebo) [27].

Nel più recente degli studi condotti su abciximab in associazione alla PCI, ISAR$\operatorname{REACT}(\mathrm{n}=2159)$ la somministrazione del farmaco in pazienti a basso rischio (erano esclusi i pazienti con sindromi coronariche acute, diabete, infarto miocardico negli ultimi 14 giorni e quelli con trombo visibile) non è stata in grado 
di migliorare gli esiti rispetto al placebo (endpoint triplo è stato raggiunto dal $4,0 \%$ dei pazienti placebo e nel $4,2 \%$ dei pazienti placebo) [28].

Nello studio TARGET, il profilo di efficacia e sicurezza dell' abciximab (bolo $0,25 \mathrm{mg} / \mathrm{kg}+$ infusione $0,125 \mu \mathrm{g} / \mathrm{kg} / \mathrm{min}$ per 12 ore) nelle PCI è stato confrontato con quello di tirofiban (bolo $10 \mu \mathrm{g} / \mathrm{kg}+$ infusione $0,15 \mu \mathrm{g} / \mathrm{kg} / \mathrm{min}$ per $18-24$ ore) in 2398 pazienti. Nonostante lo studio fosse stato disegnato per dimostrare la non inferiorità di tirofiban, questo obiettivo non è stato raggiunto: endpoint triplo di infarto morte o rivascolarizzazione del vaso target è stato raggiunto dal 7,6\% dei pazienti tirofiban contro il $6,0 \%$ dei pazienti abciximab ( RR 1,26, $\mathrm{p}=0,038$ per la superiorità di abciximab) [29].

\section{Profilo di tollerabilità e sicurezza}

I principali effetti avversi degli inibitori GP IIb/IIIa sono strettamente legati al meccanismo d'azione e sono rappresentanti da emorragie e trombocitopenia.

Nei due grandi trial PURSUIT e IMPACT II, con un totale di oltre 14.000 pazienti arruolati, l'incidenza di emorragie maggiori è risultata leggermente aumentata nei pazienti trattati con eptifibatide, a basse (135/0,5 o 0,75) o alte dosi (180/1,3 o 2): l'emorragia, principalmente a carico dell'accesso vascolare femorale, si è verificata nel 5-11\% dei casi con eptifibatide, contro il 5-9\% del placebo [13,24,30]. Nell'ESPRIT l'incidenza di emorragie maggiori e minori è stata del $1,3 \%$ e $3 \%$, rispettivamente, nel gruppo eptifibatide, contro lo $0,4 \%$ e $2 \%$ dei pazienti assegnati al placebo [22].

Con tirofiban, l'incidenza di emorragie minori e maggiori osservata nei trial clinici in cui è stato utilizzato in associazione ad aspirina ed eparina è stata del 10,5-12\% e 1,4-2,2\%, rispettivamente [31].

Episodi emorragici maggiori si verificano fino nel $14 \%$ dei pazienti sottoposti a PCI che ricevono un'iniezione di abciximab immediatamente prima dell'intervento, mentre il sanguinamento non rappresenta un seria complicazione se il farmaco viene somministrato 18-24 ore prima [32]. Nell'EPIC, episodi emorragici maggiori si sono verificati nel $10,6 \%$ dei pazienti trattati con abciximab con-

\begin{tabular}{lcc}
\hline \multicolumn{1}{c}{ Farmaco } & $\begin{array}{c}\text { Abciximab vs } \\
\text { Placebo }\end{array}$ & $\begin{array}{c}\text { Eptifibatide o Tirofiban } \\
\text { vs Placebo }\end{array}$ \\
\hline $\begin{array}{l}\text { Trombocitopenia moderata } \\
(\mathrm{PLT}<50,000 / \mu \mathrm{L})\end{array}$ & $\begin{array}{c}4,2 \% \text { vs } 2 \% * \\
\text { RR } 2,13\end{array}$ & $4,6 \%$ vs $4,6 \%$ \\
$\begin{array}{l}\text { Trombocitopenia grave } \\
(\mathrm{PLT}<20,000 / \mu \mathrm{L})\end{array}$ & $\begin{array}{c}\text { RR } 0,99 \\
\text { vs } 0,4 \% * *\end{array}$ & $0,3 \%$ vs $0,2 \%$ \\
\hline
\end{tabular}

\section{Tabella II}

Associazione della terapia a base di GPIIb/IIla con lo sviluppo di trombocitopenia

$\mathrm{p}<0,001$ per abciximab vs placebo; ** $\mathrm{p}<0,01$ per abciximab vs placebo tro il $3,3 \%$ di quelli che hanno ricevuto placebo [33].

L'utilizzo degli inibitori GP IIb/IIIa nelle sindromi coronariche acute o nelle PCI non è associato in maniera statisticamente significativa ad un aumento delle emorragie intracraniche, secondo i risultati di una meta-analisi di 12 grandi trial (abciximab 4, eptifibatide 3, lamifiban 1, tirofiban 4): il tasso è risultato simile tra i 15.850 pazienti trattati con eparina e GP IIb/IIIa inibitori e i 12.039 soggetti che hanno ricevuto eparina e placebo $(0,12 \%$ versus $0,09 \%$; RR 1,3; $\mathrm{p}=0,59)$. L'incidenza di emorragia intracranica è comunque risultata superiore nei pazienti trattati, il che non permette di escludere che esista una debole associazione con l'evento; analisi per sottogruppi hanno anche indicato che non è possibile escludere un eccesso di rischio nei pazienti con sindromi coronariche acute e in quelli trattati con abciximab [34].

L'associazione della terapia a base di GPIIb/ IIIa con lo sviluppo di trombocitopenia è stato analizzato da Dasgupta et al. in un'analisi aggregata dei risultati di 8 grandi trial, rivelando che l'utilizzo di abciximab è associato a un maggior rischio di sviluppare questa complicazione rispetto a eptifibatide o tirofiban (Tabella II) [35].

\section{Linee guida: inibitori GPIIb/IIIa e PCI}

Data la complessità della materia e la ricchezza dei dati disponibili in letteratura, presentata solo in piccola parte nelle sezioni precedenti, società scientifiche internazionali (ESC, ACC, AHA) hanno indicato delle linee guida per la gestione dell' angina instabile, degli infarti non-Q e per l'esecuzione delle PCI [36-38]. Questi documenti, aggiornati periodicamente, raccomandano i comportamenti da tenere sulla base dei risultati dei trial clinici randomizzati e riportano i pareri degli esperti su argomenti ancora controversi. Le raccomandazioni che riguardano gli inibitori GPIIb/IIIa sono contenute nelle linee guida ACCP (American College of Chest Physicians) ed ESC (European Society of Cardiology) sulle PCI.

Le linee guida ACCP raccomandano l'utilizzo di un inibitore GPIIb/IIIa (abciximab o eptifibatide) in tutti i pazienti sottoposti a PCI, particolarmente per quelli a rischio elevato; nelle PCI eseguite per infarti con sopraslivellamento del tratto ST, abciximab è da preferirsi a eptifibatide. Tirofiban non è consigliato nelle PCI [36].

Nelle PCI eseguite nei pazienti con angina stabile, le linee guida ESC raccomandano di valutare l'opportunità di somministrare un inibitore GPIIb/IIIa caso per caso, riservandola ai pazienti a rischio più elevato (depressione ST, elevati livelli enzimatici, instabilità emodinamica). 
Nei pazienti ad alto rischio in cui si prevede l'esecuzione dell' angiografia entro 2,5 ore, viene raccomandata la somministrazione di abciximab o eptifibatide; se invece si prevede di eseguire l'angiografia entro 48 ore sono raccomandati per la terapia iniziale (upstream) eptifibatide o tirofiban.

Nelle PCI eseguite in seguito a infarto miocardico acuto (con sopraslivellamento del tratto ST), infine, l'unico agente inibitore GPIIb/ IIIa raccomandato è abciximab [37].

\section{COSTO/EFFICACIA DEGLI INIBITORI GPIIB/IIIA}

\section{Trattamento upstream}

Lo studio PURSUIT è stato studiato dal punto di vista farmacoeconomico sia secondo i criteri del sistema statunitense che di quello europeo.

L'analisi farmacoeconomica del PURSUIT condotta negli Usa è stata sviluppata in due parti: la prima, di tipo osservazionale, condotta in parallelo alla raccolta dei dati clinici, ha comparato i costi ospedalieri associati all'intervento e i costi sanitari diretti in cui sono incorsi i circa 3500 pazienti nei 6 mesi successivi, mentre la seconda parte ha stimato la costo/efficacia e la costo/utilità a lungo termine di eptifibatide proiettando costi e benefici attesi per l'intera sopravvivenza residua del campione mediante tecniche di modellizzazione.

Per lo studio sui costi a breve termine sono state misurate le risorse ospedaliere consumate durante il ricovero indice (quello che ha condotto all'inclusione nello studio) e le risorse sanitarie complessivamente assorbite nei sei mesi successivi. Tali risorse sono quindi state monetizzate con prezzi e tariffe vigenti nel 1996 , evidenziando la sovrapponibilità tra la spesa sanitaria complessiva nei due gruppi. La riduzione del 3,5\% nell'incidenza di morte o infarto non fatale è stata dunque ottenuta senza incremento di spesa, con l'esclusione del costo farmaceutico di eptifibatide, pari a circa 1200 US\$ a paziente (Tabella III).

Vista l'assenza di dati oltre i 6 mesi dall'evento indice, la valutazione di costo/efficacia e costo/utilità di eptifibatide nel lungo termine ha richiesto la costruzione di un modello analitico, basato sui dati registrati e su alcune assunzioni, in particolare relative all'effetto della prevenzione di infarti miocardici non fatali sulla sopravvivenza complessiva. Il valore prognostico degli IMA non fatali è stato stimato come una riduzione approssimativa dell'aspettativa di vita di 2 anni, sulla base di un modello a regressione multipla alimentato da dati di pazienti contenuti in un vasto database clinico. Nello scenario di base, il modello ha stimato che con eptifibatide ogni paziente ottiene in media un guadagno in sopravvivenza

\begin{tabular}{|c|c|c|}
\hline & $\begin{array}{l}\text { Eptifibatide } \\
(n=1754)\end{array}$ & $\begin{array}{l}\text { Placebo } \\
(n=1765)\end{array}$ \\
\hline \multicolumn{3}{|l|}{ Ricovero indice } \\
\hline \multicolumn{3}{|l|}{ Consumo di risorse sanitarie (\%) } \\
\hline Cateterismo cardiaco & 85 & 85 \\
\hline Intervento percutaneo & 35 & 36 \\
\hline Bypass coronarico & 20 & 20 \\
\hline \multicolumn{3}{|l|}{ Durata della degenza (giorni) } \\
\hline Unità coronarica & $3,7 \pm 3,8$ & $3,7 \pm 4,0$ \\
\hline Totale & $6,5 \pm 5,2$ & $6,5+5,4$ \\
\hline \multicolumn{3}{|l|}{ Costi sanitari* (US\$) } \\
\hline Ospedalieri & $12.420 \pm 9.194$ & $12.617+10.019$ \\
\hline Personale medico & $2.309 \pm 1.793$ & $2.340 \pm 1.890$ \\
\hline Totale & $14.729 \pm 10.825$ & $14.957 \pm 11.744$ \\
\hline \multicolumn{3}{|l|}{ Follow-up (6 mesi) } \\
\hline \multicolumn{3}{|l|}{ Consumo di risorse sanitarie (\%) } \\
\hline Cateterismo cardiaco & 14 & 14 \\
\hline Intervento percutaneo & 7 & 7 \\
\hline Bypass coronarico & 4 & 4 \\
\hline \multicolumn{3}{|l|}{ Durata della degenza (giorni) } \\
\hline Unità coronarica & $0,6 \pm 2,3$ & $0,6 \pm 2,4$ \\
\hline Totale & $2,2 \pm 6,2$ & $2,3 \pm 6,3$ \\
\hline \multicolumn{3}{|l|}{ Costi sanitari del follow-up (US\$) } \\
\hline Ricoveri + personale medico & $3.727 \pm 8.878$ & $3.871 \pm 9.430$ \\
\hline $\begin{array}{l}\text { Costi sanitari complessivi nei } \\
6 \text { mesi * (US\$) }\end{array}$ & $18.456 \pm 13.886$ & $18.828 \pm 15.185$ \\
\hline
\end{tabular}

Tabella III

Valutazione economica del braccio statunitense dello studio PURSUIT: formazione dei costi

* Escluso il costo del farmaco

di 0,111 anni ( 15,95 vs 14,84$)$, a un costo incrementale di 1217 US\$ (corrispondente al prezzo del farmaco, assumendo l'assenza di differenze significative nelle altri voci di costo). Attualizzando l'entità dei costi e dei benefici con un tasso annuo di sconto del $3 \%$, è stato ottenuto un rapporto di costo/efficacia incrementale di 16491 US\$/anno di vita guadagnato. L'analisi di costo/utilità si è basata sui valori di preferenza ottenuti con la tecnica del time-trade off (al paziente viene richiesto di scambiare 10 anni nell'attuale stato di salute con un determinato numero di anni di vita in ottima salute) da circa 2000 pazienti del US PURSUIT, e ha fornito valori oscillanti introno ai 20000 US\$/QALY. I risultati ricavati con le ipotesi di partenza sono stati sottoposti ad approfondite analisi di sensibilità, per verificare la dipendenza dei valori calcolati dalle assunzioni postulate sui parametri più incerti del modello, ottenendo rapporti di costo/efficacia incrementale oscillanti tra circa $10000 \mathrm{e}$ 35000 US\$/anno di vita guadagnato e costo/ utilità fino a 25000 US\$/QALY [39] .

L'analisi economica dei risultati ottenuti nel braccio europeo è avvenuta anch'essa in due 


\section{Tabella IV \\ PCl: costo farmaceutico per evento evitato a 30 giorni (in un paziente di $84 \mathrm{~kg}$ - valore medio ESPRIT)}

\begin{tabular}{|c|c|c|}
\hline Farmaco & Abciximab & Eptifibatide \\
\hline Schema posologico & $\begin{array}{l}\text { Bolo 0,25 mg/kg }+ \\
\text { infusione } 0,125 \mu \mathrm{g} / \mathrm{kg} / \mathrm{min}\end{array}$ & $\begin{array}{l}\text { Bolo } 180 \mu \mathrm{g} / \mathrm{kg}+\text { infusione } 2,0 \mu \mathrm{g} / \mathrm{kg} / \mathrm{min}+ \\
\text { altro bolo } 180 \mu \mathrm{g} / \mathrm{kg} 10 \mathrm{~min} \text { dopo il primo }\end{array}$ \\
\hline Durata dellinfusione & 12 h (EPILOG) & 18,3 h (Media ESPRIT) \\
\hline Confezioni necessarie & $3 \mathrm{fl} 5 \mathrm{ml} 10 \mathrm{mg}$ & $2 \mathrm{f} 10 \mathrm{ml} 2 \mathrm{mg} / \mathrm{ml}+3 \mathrm{fl} 100 \mathrm{ml} 0,75 \mathrm{mg} / \mathrm{ml}$ \\
\hline Prezzo confezioni al SSN & 256,83 euro & $\begin{array}{l}20,41 \text { euro (fiala endovena) } \\
64,46 \text { euro (flacone infusione) }\end{array}$ \\
\hline Costo totale & 770,49 euro & 234,2 euro \\
\hline
\end{tabular}

fasi. Nella prima, che aveva come obiettivo la quantificazione delle risorse consumate mediamente dai pazienti nei due gruppi di trattamento, sono stati raccolti i dati relativi al consumo di risorse di tutti i pazienti arruolati in Europa (circa 3.700). Tali risorse sono state successivamente monetizzate in base ai prezzi e alle tariffe vigenti nei singoli paesi, con risultati abbastanza omogenei: in nessun setting sanitario la differenza di costi è risultata statisticamente significativa, per quanto costantemente superiore per eptifibatide (range: 211Euro, Olanda - 399 Euro, Spagna).

La proiezione dei risultati ottenuti nei sei mesi di follow-up in termini di sopravvivenza complessiva si è avvalsa del medesimo modello utilizzato nello studio americano, sostituendo gli outcomes clinici dei pazienti statunitensi con quelli registrati in Europa e ottenendo una stima di 0,029 anni di vita guadagnati. Nello scenario di base, dopo attualizzazione di costi e benefici, vengono riportati valori di costo/efficacia incrementale oscillanti tra 9.600 e 18.100 euro per anno di vita salvato, a seconda della nazione. La variazione delle stime sui parametri più incerti condotte nell'analisi di sensibilità hanno sostanzialmente confermato la robustezza dei risultati ottenuti, fornendo stime variabili tra circa 7.000 e circa 23.000 euro per anno di vita salvata [40].

La farmacoeconomia di tirofiban è stata valutata nello studio RESTORE: l'analisi economica ha rivelato costi sanitari cumulativi a 6 mesi molto simili nei due gruppi di trattamento (12402 \pm 6147 US\$ nel gruppo placebo e 12446 \pm 5814 US\$ nel gruppo tirofiban, $\mathrm{p}=0,87$ ). Nonostante l'esigua differenza di costo tra gruppi, il rapporto di costo/efficacia calcolato è risultato relativamente alto, circa 37.000 US\$ per anno di vita salvato, per la ridotta dimensione del beneficio clinico ottenuto [41, 42].

\section{PCI}

I risultati dell'ESPRIT sono stati valutati dal punto di vista farmacoeconomico, con particolare attenzione per i costi sostenuti nel corso del ricovero per PCI. I risultati di questo lavoro indicano che oltre il $40 \%$ dei costi di acquisizione del farmaco (circa 500 US\$ per intervento) sono stati compensati già nel corso del ricovero, gra- zie alla riduzione dei costi legati alla PCI e alle sue complicazioni ischemiche [43].

Numerosi sono gli studi di valutazione farmacoeconomica condotti sulla base dei trial clinici che hanno valutato l'utilizzo di abciximab; in particolare, la valorizzazione degli studi EPIC ed EPILOG ha dimostrato significative differenze di costo rispetto al placebo, variabili da circa 300 a 1250 US\$ per paziente trattato [42].

La Tabella IV valorizza in termini monetari gli schemi posologici di abciximab ed eptifibatide, raccomandati per i pazienti sottoposti a PCI dalle linee guida succitate, utilizzando il prezzo al SSN.

Il costo di acquisto della quantità di abciximab necessaria in un intervento di PCI è più di tre volte superiore a quello di eptifibatide nella medesima indicazione. È chiaro che la spesa farmaceutica è solo una delle componenti, e non la principale, del costo complessivo associato alla gestione di questi pazienti, per cui differenze di outcome clinico si possono riflettere in differenze sul costo complessivo.

Lo studio PRICE (Prairie ReoPro Versus Integrilin Cost Evaluation) ha valutato gli aspetti farmacoeconomici della somministrazione di abciximab o eptifibatide nei pazienti sottoposti a PCI [44]. Lo studio PRICE è stato condotto su 320 pazienti consecutivi sottoposti a PCI d'elezione (con stent impiantato nel $91 \%$ dei pazienti), randomizzati in doppio cieco a ricevere abciximab (bolo $0,25 \mu \mathrm{g} / \mathrm{kg}+$ infusione di 0,125 $\mu \mathrm{g} / \mathrm{kg} / \mathrm{min}$ per 12 ore) o eptifibatide (bolo 180 $\mu \mathrm{g} / \mathrm{kg}+$ infusione $2 \mu / \mathrm{kg} / \mathrm{min}$ per $18-24$ ore). I risultati clinici non hanno mostrato differenze significative tra i due gruppi di trattamento, né in termini di efficacia (endpoint combinato a 30 giorni $5,6 \%$ vs $6,3 \%$ per abciximab ed eptifibatide, rispettivamente, $\mathrm{p}=0,95$ ), né di sicurezza (emorragie maggiori $3,1 \%$ vs $1,9 \%$ per abciximab ed eptifibatide, rispettivamente, $\mathrm{p}=0,72$ ). A fronte dell'equivalenza dei risultati clinici, il costo globale mediano (inclusi cateterizzazione coronarica, servizi di cardiologia, laboratorio e costi farmaceutici) associato al trattamento con eptifibatide, è risultato significativamente inferiore rispetto a quello associato con abciximab ( $\$ 7207$ vs $\$ 8336$, $\mathrm{p}=0.009$ ). La maggior parte della differenza è risultata dovuta al costo farmaceutico in sé [44]. 


\begin{tabular}{lccc}
\multicolumn{1}{c}{ Farmaco } & Eptifibatide & Abciximab & Delta EPT-ABC(p) \\
\hline Efficacia: incidenza endpoint & $6,3 \%$ & $5,6 \%$ & $+0,7 \%(\mathrm{NS}: 0,95)$ \\
Tollerabilità: emorragie maggiori & $3,1 \%$ & $1,9 \%$ & $-1,2 \%(\mathrm{NS}: 0,72)$ \\
Costi ospedalieri & $\$ 7207$ & $\$ 8336$ & $-\$ 1129(0,009)$ \\
\hline
\end{tabular}

Il costo, farmaceutico o totale che sia, è comunque solo una delle componenti di ogni valutazione farmacoeconomica completa, che deve necessariamente prendere anche in considerazione la differenza di esiti clinici, per calcolare dei rapporti di costo/efficacia o costo/utilità, a seconda che venga considerata anche la qualità di vita. Alcuni degli studi brevemente delineati in precedenza sono stati anche accompagnati dalla stima di questi rapporti, la cui esposizione dettagliata non è possibile in questo contesto. A titolo indicativo e con l'avvertimento di utilizzare cautela nella loro interpretazione, in quanto trattasi di dati ottenuti in paesi differenti su pazienti con caratteristiche diverse, in Tabella VI presentiamo i risultati ottenuti negli studi di valutazione del PURSUIT. Lo studio PURSUIT è particolarmente adatto alla valutazione economica perché è il trial con la maggiore casistica di pazienti con sindrome coronarica acuta attualmente disponibile e per il tipo di disegno flessibile, che non imponeva un particolare protocollo di gestione clinica, per cui le risorse consumate riflettono più fedelmente la prassi clinica rispetto a disegni più rigidi, e per la disponibilità di dati stratificati per nazione $\mathrm{e}$ per sottogruppi, che hanno consentito di esplorare molti differenti scenari $[42,45]$.

Questi valori, così come quelli calcolati per abciximab nelle PCI (2875 -14765 US\$), rientrano ampiamente entro i limiti convenzionalmente fissati come soglia della disponibilità a pagare per i benefici clinici delle società avanzate, fissata intorno ai 50.000 euro per QALY (quality-adjusted life year, anno di vita ponderato per la qualità) guadagnato [42].

\section{CONCLUSIONI}

I tre inibitori del recettore glicoproteico GPIIb/ IIIa, per quanto attivi sul medesimo meccanismo biochimico, differiscono tra loro per struttura chimica, campo di applicabilità e implicazioni economiche. Il corpo dell' evidenza scientifica a supporto della loro utilità nella sindrome coronarica acuta senza sopraslivellamento del tratto ST è molto solido, per quanto il profilo di efficacia delle singole molecole sia funzione delle caratteristiche farmacodinamiche della molecola e delle caratteristiche cliniche del paziente trattato, tanto che le più recenti linee guida suggeriscono precisi usi terapeutici per ciascuna molecola.

Tirofiban appare avere il range di indicazioni più ridotto, essendo raccomandato solo nei pazienti ad alto rischio con in cui si prevede l'esecuzione dell' angiografia entro le 48 ore, seguita se necessario dalla PCI. Abciximab è indicato per quei casi in cui viene eseguita l'angiografia entro le 2,5 ore o nei casi di PCI senza precedente copertura in upstream con altri GP IIb IIIa. Eptifibatide, infine, è raccomandata sia quando si prevede di eseguire l'angiografia (seguita se necessario dalla PCI) entro le 2,5 ore sia entro le 48 ore, sia nei casi di PCI senza precedente copertura in upstream con altri GP IIb IIIa.

Le raccomandazioni fornite dalle società scientifiche si basano sull'analisi dei lavori scientifici; ciononostante, l'utilizzo clinico di queste molecole è ancora piuttosto ridotto, in gran parte a causa del loro elevato costo di acquisizione [42]. Gli studi farmacoeconomici condotti sul loro utilizzo, tuttavia, hanno dimostrato che queste molecole, quando impiegate in accordo alle raccomandazioni e con gli schemi posologici approvati, producono indiscussi benefici clinici a un costo che è in linea o inferiore a quello di altre tecnologie sanitarie ampiamente accettate dalle nostre società avanzate. Si tratta inoltre di farmaci salvavita, per cui la loro prescrizione non si può basare unicamente su considerazioni relative al costo di acquisto, ma deve necessariamente fondarsi anche su valutazioni etiche. Per di più, la valutazione dei soli costi di acquisizione è impropria anche dal punto di vista economico, come indicato dagli studi farmacoeconomici condotti sul loro utilizzo, in quanto una quota dell'eccesso di spesa farmaceutica è compensata dalla riduzione dei costi ospedalieri sostenuti per la gestione dei pazienti con sindrome coronarica acuta.

\section{Tabella V}

Studio PRICE: risultati principali

\begin{tabular}{lc}
\hline \multicolumn{1}{c}{ Studio } & Costo/anno di vita salvato \\
\hline Sottostudio US PURSUIT & 16.491 US\$ \\
Sottostudio Europeo PURSUIT, valori medi & $9.603-18.115$ Euro \\
$\begin{array}{l}\text { Sottostudio Europeo PURSUIT, valori nei } \\
\text { paesi con basso tasso di PCI }\end{array}$ & $3.329-10.079$ Euro \\
$\begin{array}{l}\text { Sottostudio Europeo PURSUIT, valori nei } \\
\text { paesi con alto tasso di PCI }\end{array}$ & $17.089-24.099$ Euro \\
\hline $\begin{array}{l}\text { Tabella VI } \\
\text { Rapporti di costo/efficacia incrementale calcolati in base allo studio PURSUIT }\end{array}$
\end{tabular}




\section{BIBLIOGRAFIA}

1. Simoons ML, Jan de Boer M, van den Brand MJBM et al: Randomized trial of a GPIIb/IIIa platelet receptor blocker in refractory unstable angina. Circulation 1994; 89:596-603

2. Saucedo JF, Lui HK, Garza L, Guerra GJ, Jacoski MV, Jennings LK. Comparative pharmacodynamic evaluation of eptifibatide and abciximab in patients with non-ST-segment elevation acute coronary syndromes: the TAM2 study.J Thromb Thrombolysis. 2004 Oct;18(2):67-74.

3. Kereiakes DJ, Kleiman NS, Ambrose J et al: Randomized, double-blind, placebo-controlled dose-ranging study of tirofiban (MK-383) platelet IIb/IIIa blockade in high risk patients undergoing coronary angioplasty. J Am Coll Cardiol 1996; 27:536-542

4. Barrett JS, Murphy G, Peerlinck K et al: Pharmacokinetics and pharmacodynamics of MK-383, a selective nonpeptide platelet glycoprotein-IIb/IIIa receptor antagonist, in healthy men. Clin Pharm Ther 1994; 56:37-388.

5. Kereiakes DJ, Broderick TM, Roth EM, et al.:Time course, magnitude, and consistency of platelet inhibition by abciximab, tirofiban, or eptifibatide in patients with unstable angina pectoris undergoing percutaneous coronary intervention. Am J Cardiol. 1999 Aug 15;84(4):391-5.

6. Goldschmidt-Clermont PJ, Schulman SP, Bray PF et al: Refining the treatment of women with unstable angina - a randomized, double-blind, comparative safety and efficacy evaluation of Integrelin(TM) versus aspirin in the management of unstable angina. Clin Cardiol 1996; 19:869-874.

7. Tcheng JE, Harrington RA, Kottke-Marchant K et al: Multicenter, randomized, double-blind placebo-controlled trial of the platelet integrin glycoprotein IIb/IIIa blocker Integrelin(TM) in elective coronary intervention. Circulation 1995; 91:2151-2157.

8. Saucedo JF, Garza L, Wolford DC, Cook SL, et al: Comparative pharmacodynamic evaluation of eptifibatide and tirofiban $\mathrm{HCl}$ in patients undergoing percutaneous coronary intervention (the TAM1 Study).Am J Cardiol. 2004 May 15;93(10):1279-82

9. The PURSUIT Trial Investigators. Inhibition of platelet glycoprotein IIb/IIIa with eptifibatide in patients with acute coronary syndromes. N Engl J Med. 1998 Aug 13;339(7):436-43.

10. Adgey AAJ: An overview of the results of clinical trials with glycoprotein IIb/IIIa inhibitors. Eur Heart J 1998; 19(suppl D):D10-D21

11. Harrington RA: Design and methodology of the PURSUIT trial: evaluating eptifibatide for acute ischemic coronary syndromes. Am J Cardiol 1997; 80(4A):34B-38B

12. Fintel DJ, Ledley GS. Management of patients with non-ST-segment elevation acute coronary syndromes: insights from the PURSUIT trial. Clin Cardiol. 2000 Sep;23 Suppl 5:V1-12.

13. Lincoff AM, Harrington RA, Califf RM, et al.:Management of patients with acute coronary syndromes in the United States by platelet glycoprotein IIb/IIIa inhibition. Insights from the platelet glycoprotein IIb/IIIa in unstable angina: receptor suppression using integrilin therapy (PURSUIT) trial. Circulation. 2000 Sep 5;102(10):1093-100

14. Ronner E, Boersma E, Akkerhuis KM, et al.:Patients with acute coronary syndromes without persistent ST elevation undergoing percutaneous coronary intervention benefit most from early intervention with protection by a glycoprotein IIb/IIIa receptor blocker. Eur Heart J. 2002 Feb;23(3):239-46

15. Dyke CM, Bhatia D, Lorenz TJ, et al.:Immediate coronary artery bypass surgery after platelet inhibition with eptifibatide: results from PURSUIT. Ann Thorac Surg. 2000 Sep;70(3):866-71

16. Tcheng JE, Strony J, Lorenz TJ, O’Shea JC: ESPRIT in context: pharmacology matters! Eur Heart J. 2001 Nov;22(21):1965-7.

17. Phillips DR, Tent W, Arfsten A et al: Effect of Ca2+ on GP IIb-IIIa interactions with Integrilin(TM): enhanced GP IIb-III binding and inhibition of platelet aggregation by reductions in the concentration of ionized calcium in plasma anticoagulated with citrate. Circulation 1997; 96:1488-1494.

18. The platelet receptor inhibition in ischemic syndrome management in patients limited by unstable signs and symptoms (PRISM-PLUS) study investigators: Inhibition of the platelet glycoprotein IIb/IIIa receptor with tirofiban in unstable angina and non-Q-wave myocardial infarction. N Engl J Med 1998: 338(21):1488-1497.

19. The platelet receptor inhibition in ischemic syndrome management (PRISM) study investigators: A comparison of aspirin plus tirofiban with aspirin plus heparin for unstable angina:. N Engl J Med 1998; 338(21):1498-1505.

20. Chesebro JH \& Badimon JJ: Platelet glycoprotein IIb/IIIa receptor blockade in unstable coronary disease. $\mathrm{N}$ Engl J Med 1998; 338(21):1539-1541. 
21. The GUSTO IV-ACS Investigators: Effect of glycoprotein IIb/IIIa receptor blocker abciximab on outcome in patients with acute coronary syndromes without early coronary revascularisation: the GUSTO IV-ACS randomised trial;. Lancet 2001; 357:1915-1924.

22. ESPRIT Investigators. Enhanced Suppression of the Platelet IIb/IIIa Receptor with Integrilin Therapy:.Novel dosing regimen of eptifibatide in planned coronary stent implantation (ESPRIT): a randomised, placebo-controlled trial.Lancet. 2000 Dec 16;356(9247):2037-44.

23. O'Shea JC, Hafley GE, Greenberg S et al: Platelet glycoprotein IIb/IIIa integrin blockade with eptifibatide in coronary stent intervention; the ESPRIT trial: a randomized controlled trial. JAMA 2001; 285:2468-2473.

24. The IMPACT II investigators: Randomised placebo-controlled trial of effect of eptifibatide on complication of percutaneous coronary intervention: IMPACT II. Lancet 1997; 349:1422-1428.

25. The RESTORE Investigators: Effects of platelet glycoprotein IIb/IIIa blockade with tirofiban on adverse cardiac events in patients with unstable angina or acute myocardial infarction undergoing coronary angioplasty.Circulation. 1997 Sep 2;96(5):1445-53.

26. Valgimigli M, Percoco G, Barbieri Det al.: The additive value of tirofiban administered with the high-dose bolus in the prevention of ischemic complications during high-risk coronary angioplasty: the ADVANCE Trial. J Am Coll Cardiol. 2004 Jul 7;44(1):14-9

27. The EPILOG Investigators: Platelet glycoprotein IIb/IIIa receptor blockade and low-dose heparin during percutaneous coronary revascularization.. N Engl J Med. 1997 Jun 12;336(24):1689-96.

28. Kandzari DE, Berger PB, Kastrati A, et al: Influence of treatment duration with a 600-mg dose of clopidogrel before percutaneous coronary revascularization. J Am Coll Cardiol. 2004 Dec 7;44(11):2133-6.

29. Topol EJ, Moliterno DJ, Herrmann HC et: Comparison of two platelet glycoprotein IIb/IIIa inhibitors, tirofiban and abciximab, for the prevention of ischemic events with percutaneous coronary revascularization. New Engl J Med 2001;344:1888-1894

30. Blankenship JC: Bleeding complications of glycoprotein IIb/IIIa receptor inhibitors. Am Heart J 1999; 138:S287-S296.

31. Aggrastat @: Riassunto delle caratteristiche del prodotto, Ottobre 1999

32. Reopro ®: Riassunto delle caratteristiche del prodotto, Giugno 1996

33. The EPIC Investigators: Use of a monoclonal antibody directed against the platelet glycoprotein IIb/IIIa receptor in high-risk coronary angioplasty. N Engl J Med 1994; 330:956-961.

34. Memon MA, Blankenship JC, Wood GC, et al.:Incidence of intracranial hemorrhage complicating treatment with glycoprotein IIb/III receptor inhibitors: a pooled analysis of major clinical trials. Am J Med. 2000 Aug 15;109(3):213-7.

35. Dasgupta H, Blankenship JC, Wood GC et al: Thrombocytopenia complicating treatment with intravenous glycoprotein IIb/IIIa receptor inhibitors: A pooled analysis. Am Heart J 2000; 140:206-211

36. Popma JJ, Berger P, Ohman EM, et al.: Antithrombotic therapy during percutaneous coronary intervention: the Seventh ACCP Conference on Antithrombotic and Thrombolytic Therapy. Chest. 2004 Sep;126(3 Suppl):576S-599S.

37. Silber S, Albertsson P, Aviles FF, et al.: Guidelines for percutaneous coronary interventions. The Task Force for Percutaneous Coronary Interventions of the European Society of Cardiology. Eur Heart J. 2005 Apr;26(8):804-47.

38. National Institute for Clinical Excellence: Technology Appraisal Guidance - No. 47: Guidance on the use of glycoprotein IIb/IIIa inhibitors in the treatment of acute coronary syndromes . NICE, London, issued September 2002, reviewed July 2005.

39. Mark DB, Harrington RA, Lincoff AM,et al.: Cost-effectiveness of platelet glycoprotein IIb/IIIa inhibition with eptifibatide in patients with non-ST-elevation acute coronary syndromes. Circulation. 2000 Feb 1;101(4):366-71.

40. Brown RE, Henderson RA, Koster D, et al.: Cost effectiveness of eptifibatide in acute coronary syndromes; an economic analysis of Western European patients enrolled in the PURSUIT trial. Eur Heart J. 2002 Jan;23(1):50-8

41. Weintraub WS, Culler S, Boccuzzi SJ, et al.: Economic impact of GPIIB/IIIA blockade after high-risk angioplasty: results from the RESTORE trial. J Am Coll Cardiol. 1999 Oct;34(4):1061-6

42. Hillegass WB, Newman AR, Raco DL. Glycoprotein IIb/IIIa receptor therapy in percutaneous coronary intervention and non-ST-segment elevation acute coronary syndromes. Estimating the economic implications. Pharmacoeconomics. 2001 Jan;19(1):41-55.

43. Cohen DJ, O'Shea JC, Pacchiana CM, et al. In-hospital costs of coronary stent implantation with and without eptifibatide (the ESPRIT trial). Am J Cardiol 2002 Jan 1; 89 (1): 61-4 
44. PRICE Investigators.: Comparative 30-day economic and clinical outcomes of platelet glycoprotein IIb/IIIa inhibitor use during elective percutaneous coronary intervention: Prairie ReoPro versus Integrilin Cost Evaluation (PRICE) trial. Am Heart J 2001 Mar; 141 (3): 402-9

45. Plosker GL, Ibbotson T. Eptifibatide: a pharmacoeconomic review of its use in percutaneous coronary intervention and acute coronary syndromes. Pharmacoeconomics. 2003;21(12):885-912. 